\title{
On the Generalised Character Compatibility Problem for Non-branching Character Trees
}

\author{
Ján Maňuch, Murray Patterson, and Arvind Gupta \\ School of Computing Science, Simon Fraser University, 8888 University Drive, \\ Burnaby, British Columbia, Canada
}

\begin{abstract}
In [3], the authors introduced the Generalised Character Compatibility Problem as a generalisation of the Perfect Phylogeny Problem for a set of species. This generalised problem takes into account the fact that while a species may not be expressing a certain trait, i.e., having teeth, its DNA may contain data for this trait in a non-functional region. The authors showed that the Generalised Character Compatibility Problem is NP-complete for an instance of the problem involving five states, where the characters' state transition trees are branching. They also presented a class of instances of the problem that is polynomial-time solvable. The authors posed an open problem about the complexity of this problem when no branching is allowed in the character trees. They answered this question in 2, where they showed that for an instance in which each character tree is $0 \rightarrow 1 \rightarrow 2$ (no branching), and only the states $\{1\},\{0,2\},\{0,1,2\}$ are allowed, is NP-complete. This, however, does not provide an answer to the exact question posed in [3], which allows only one type of generalised state: $\{0,2\}$, called here the BenhamKannan-Warnow (BKW) Case.

In this paper, we study the complexity of various versions of this problem with non-branching character trees, depending on the set of states allowed, and depending on the restriction on the phylogeny tree: any tree, path or single-branch tree. In particular, we show that if the phylogeny tree is required to have only one branch: (a) the problem still remains NP-complete (for instance with states $\{1\},\{0,2\},\{0,1,2\}$ ), and (b) the problem is polynomial-time solvable in the BKW Case (with states $\{0\},\{1\},\{2\},\{0,2\})$. We show the second result by unveiling a surprising connection to the Consecutive-Ones Property (C1P) Problem, used for instance, in DNA physical mapping, interval graph recognition and data retrieval.
\end{abstract}

\section{Introduction}

Constructing an evolutionary history from a set of species is the standard practice of computational evolutionary biologists [6]. A classical problem in this area is the Perfect Phylogeny Problem, the problem of trying to find a tree that explains the evolutionary relationship of a set of species. Here, each species is described by a set of characters. For a given species, a character only has a single 
state (i.e., the number of legs is four, or the eye color is blue). The Perfect Phylogeny Problem is shown to be NP-complete in 413] and solvable in polynomial time when any of the associated parameters is fixed 18911.

In 3], the authors argue why describing each species simply with characters that only have a single state for that species is not enough to accurately capture an evolutionary history from a set of species. In the human genome, only about $5 \%$ of the DNA is actually genes, the rest of it termed "junk" DNA, while some species have an even higher percentage of non-functional DNA. It can persist through evolution only because there is little or no selection pressure favoring streamlined genomes in multicelled organisms. Recent work has shown that this non-functional DNA can actually contain genes that were once active in the ancestors of a species, but are not presently expressed, perhaps due to mutations 7[10. The most striking example of this is the demonstration that embryonic chicken tissue can be induced to differentiate into a tooth structure [10]. Although modern birds are well known not to have teeth, Archaeopteryx, their ancestral form of some 100 million years ago, was toothed.

Describing species with simple characters implies that a species only contains the data for the currently expressed state of the character. To remedy this, in [3], the authors propose the concept of generalised characters. Rather than having only a single state for a character and a species, a generalised character takes on a set of states (which we call generalised states, or just states when the context is clear), where we only know that the expressed state is in this set. This captures the fact that while a species is expressing a certain trait, it may contain the genetic information to express other traits as well. In addition to this, generalised characters also incorporate knowledge about the direction of transitions between character states (a transition from a double-sided mammalian heart to a hollow muscular tube heart will never happen, for example). The set of constraints on character state transitions is represented by a rooted tree, which we call a character tree, associated with the character. Here, the direction of character state transitions is always from parent to child in the character tree.

Since this new type of character is a generalisation of the "classical" character, there is also the notion of the Perfect Phylogeny Problem when species are described by generalised characters, which is termed the Generalised Character Compatibility (GCC) Problem, as opposed to the Classical Character Compatibility Problem. The authors of [3] present a polynomial-time algorithm for the case of the GCC Problem where for each character and each species, the set of states forms a directed path in the character tree. They also show that the general case is NP-complete. They do so by showing NP-completeness for a case when each character has five states, and the characters' state transition trees are branching. However, in this setting the situation when a gene becomes hidden does not happen, therefore the authors posed an open problem about the complexity of the GCC Problem when the character trees are $0 \rightarrow 1 \rightarrow 2$ and the only states assigned to input species allowed are $\{0\},\{1\},\{2\}$ and $\{0,2\}$ (we call this the Benham-Kannan-Warnow (BKW) Case). In [2], NP-completeness for a slight variant of this case in which we also allow the "wildcard" state $\{0,1,2\}$ 
(the information about the state for a particular species is unknown) was shown. We study the complexity of various variations of the BKW Case. In particular, we show that the GCC Problem when the character trees are not branching is NPcomplete even when the phylogeny tree is required to have only one branch. We also show that the GCC Problem in the BKW Case can be solved in polynomial time when the phylogeny tree is required to have only one branch. Interestingly, the algorithm for this polynomial case is based on the algorithm for determining whether a 0-1-matrix has the Consecutive-Ones Property, used for instance, in DNA physical mapping, interval graph recognition and data retrieval [14].

The rest of this paper is organised as follows: In Sect. 2 we define the Path Triple Consistency Problem and show that it is NP-complete (a problem we use later in our proofs). We also restate in detail, the definition of the GCC Problem first defined in [3]. In Sect. 3 we show that the GCC Problem with nonbranching character trees is NP-complete, even if the solution is required to be a single-branch tree. We then consider the BKW Case in Sect. 4, and show that it is polynomial-time solvable when the solution is required to be a single-branch tree. Finally, in Sect. 5. we conclude this paper with some new open problems.

\section{Preliminaries}

\subsection{The Path Triple Consistency Problem}

The Quartet Consistency (QC) Problem was shown by Steel [13] to be NPcomplete. The QC Problem is, given a set $S=\{1, \ldots, n\}$ and a set of quartets $\left\{a_{i}, b_{i}: c_{i}, d_{i} \mid i=1, \ldots, k\right\}$, where for $i=1, \ldots, k, a_{i}, b_{i}, c_{i}, d_{i} \in S$, is there a tree $T$ on vertices $S$ such that for each $i=1, \ldots, k$, there is an edge $e_{i}$ of $T$ whose removal separates vertices $\left\{a_{i}, b_{i}\right\}$ from vertices $\left\{c_{i}, d_{i}\right\}$. That is, the solution to the QC Problem is a tree where for every quartet, there is an edge separating that quartet. Surprisingly, if we restrict this tree to being a path, the problem is still NP-complete.

Since, in this restricted version of QC Problem, the quartets must all fall on a path, a quartet of the form $a, b: c, d$ can be replaced by the three constraints $a, b: c, a, b: d$ and $c, d: a$. Thus, we can re-pose this restricted version of the QC Problem as a set of triples of this form, where they must fall on a path. We will call this the Path Triple Consistency Problem. The formal definition of the problem is as follows:

\section{Path Triple Consistency (PTC) Problem}

Input: A set $S=\{1, \ldots, n\}$ and a set of triples $\left\{a_{i}, b_{i}: c_{i} \mid i=1, \ldots, k\right\}$, where $a_{i}, b_{i}, c_{i} \in S$ for every $i=1, \ldots, k$.

Question: Is there a path (order) $P$ on vertices $S$ such that for each $i=1, \ldots, k$, there is an edge $e_{i}$ of $P$ whose removal separates vertices $\left\{a_{i}, b_{i}\right\}$ from vertex $c_{i}$.

Lemma 1. The PTC Problem is NP-complete. 
Proof. The PTC Problem is actually complementary to the Total Ordering (TO) Problem, which was shown to be NP-hard by Opatrny in 1979 [12]. The TO Problem is, given a set $Q=\{1, \ldots, n\}$ and a set of triples $\left\{a_{i}, b_{i}, c_{i} \mid i=1, \ldots, k\right\}$, where for $i=1, \ldots, k, a_{i}, b_{i}, c_{i} \in S$, is there a path (order) on $Q$ such that for each $i=1, \ldots, k$, either $a_{i}<b_{i}<c_{i}$ or $c_{i}<b_{i}<a_{i}$. It is easy to see that the NP-completeness of the TO Problem implies the NP-completeness of the PTC Problem. Given instance of TO Problem $Q=\{1, \ldots, n\}$ and $\left\{a_{i}, b_{i}, c_{i} \mid i=\right.$ $1 \ldots, k\}$, for the corresponding PTC Problem instance we let $S=Q$, and for each triple $a, b, c$ of the TO Problem instance, we introduce the triples $a, b: c$ and $c, b: a$.

\subsection{The Generalised Character Compatibility Problem}

The Generalised Character Compatibility Problem [3 is a generalization of the Perfect Phylogeny Problem [4] where, instead of classical characters (where for a given species, a character only has one state for that species), the species set $S$ is defined by a set of generalised characters. A generalised character is a pair $\hat{\alpha}=\left(\alpha, T_{\alpha}\right)$, such that:

1. $\alpha$ is a function $\alpha: S \rightarrow 2^{Q_{\alpha}}$, where $Q_{\alpha}$ denotes the set of states of $\hat{\alpha}$.

2. $T_{\alpha}=\left(V\left(T_{\alpha}\right), E\right)$ is a rooted character tree with nodes bijectively labelled by the elements of $Q_{\alpha}$.

The Generalised Character Compatibility Problem is as follows:

\section{Generalised Character Compatibility (GCC) Problem}

Input: A set $S$ of species and a set $C$ of generalised characters.

Question: Is there a rooted tree $T=\left(V_{T}, E_{T}\right)$ and a "state-choosing" function $c: V_{T} \times C \rightarrow \bigcup_{\hat{\alpha} \in C} Q_{\alpha}$ such that the following holds:

1. For each species $s \in S$ there is a vertex $v_{s}$ in $T$ such that for each $\hat{\alpha} \in C$, $c\left(v_{s}, \hat{\alpha}\right) \in \alpha(s)$.

2. For every $\hat{\alpha} \in C$ and $i \in Q_{\alpha}$, the set $\{v \in T \mid c(v, \hat{\alpha})=i\}$ is a connected component of $T$.

3. For every $\hat{\alpha} \in C$, the tree $T(\alpha)$ is an induced subtree of $T_{\alpha}$, where $T(\alpha)$ is the tree obtained from $T$ by labelling the nodes of $T$ only with their $\alpha$-states (as chosen by $c$ ), and then contracting edges having the same $\alpha$-state at their endpoints.

Essentially, the first condition states that each species is represented somewhere in the tree $T$, and the second condition states that the set of nodes labelled by a given state of a given character form a connected subtree of $T$, just as with the Classical Character Compatibility Problem. Finally, condition three states that the state transitions for each character $\hat{\alpha}$ must respect its character tree $T_{\alpha}$.

The GCC Problem is NP-complete. In particular, in 2] it was shown to be NP-complete for a case where for each species $s$ and character $\hat{\alpha}, \alpha(s) \in$ 
$\{\{1\},\{0,2\},\{0,1,2\}\}$, and $T_{\alpha}$ is $0 \rightarrow 1 \rightarrow 2$. It was also shown to be polynomialtime solvable in the case where for each species $s \in S, \alpha(s)$ is a directed path in $T_{\alpha}$ for each $\hat{\alpha}=\left(\alpha, T_{\alpha}\right) \in C[3]$. We will consider the following variants of the GCC Problem. The GCC Problem with non-branching character trees (GCC-NB Problem) is a special case of the GCC Problem in which character trees have a single branch, i.e., each character tree $T_{\alpha}$ is $0 \rightarrow 1 \rightarrow \cdots \rightarrow\left|T_{\alpha}\right|-1$. If we restrict the solution of the GCC-NB Problem (a phylogeny tree) to have only one (or two) branches starting at the root, we will call this problem the Single-Branch GCC-NB Problem (SB-GCC-NB Problem), and the Path GCC-NB (P-GCC-NB Problem), respectively. In addition, if in any of these problems, say in problem $X$, we restrict the states to be from the set $\mathcal{Q}$, we will call this problem the $\mathcal{Q}-X$ Problem.

\section{The SB-GCC-NB Problem Is NP-Complete}

We first show that the GCC-NB Problem is NP-complete even when the solution is restricted to be a single-branch tree.

Theorem 1. The $\{\{1\},\{0,2\},\{0,1,2\}\}-S B$-GCC-NB Problem is NP-complete.

Proof. Given instance of the PTC Problem $S=\{1, \ldots, n\}$, and the set of $k$ triples $\left\{a_{i}, b_{i}: c_{i}\right\}$, we let $S$ be the set of species, and let $C=\left\{\hat{\alpha}_{1}, \ldots, \hat{\alpha}_{k}\right\}$ be the set of characters, where character $\hat{\alpha}_{i}$ corresponds to triple $a_{i}, b_{i}: c_{i}$. For $\hat{\alpha}_{i} \in C$, we let $\alpha_{i}\left(a_{i}\right)=\alpha_{i}\left(b_{i}\right)=\{1\}$ and $\alpha_{i}\left(c_{i}\right)=\{0,2\}$, while for all other $s \in S \backslash\left\{a_{i}, b_{i}, c_{i}\right\}$ we let $\alpha_{i}(s)=\{0,1,2\}$.

Note that the constraints have been chosen in such a way that for each $i$, species $c_{i}$ must be before both $a_{i}$ and $b_{i}$, or after both $a_{i}$ and $b_{i}$ on the path, by choosing the $i$-th character of $c_{i}$ to be 0 or 2 , respectively. That is, the solutions to this instance of the SB-GCC-NB Problem correspond to solutions to the instance of the PTC Problem, and vice versa. The claim follows by Lemma 1 .

Next, we show that if $\mathcal{Q} \subseteq 2^{\{0, \ldots, m\}}$, the $\mathcal{Q}$-SB-GCC-NB Problem is NP-complete, then $\mathcal{Q} \cup\{\{m\}\}$-(P-)GCC-NB Problems are NP-complete.

Theorem 2. If for $\mathcal{Q} \subseteq 2^{\{0, \ldots, m\}}$, the $\mathcal{Q}-S B-G C C-N B$ Problem is NP-complete, then the $\mathcal{Q} \cup\{\{m\}\}-P-G C C-N B$ and $\mathcal{Q} \cup\{\{m\}\}-G C C-N B$ Problems are NPcomplete.

Proof. We will prove the claim by reduction from the $\mathcal{Q}$-SB-GCC-NB Problem. An instance of the SB-GCC-NB Problem can be considered as an instance of the (P-)GCC-NB Problem, provided that we can force all species to be on a single branch. This can be done easily by adding the extra species $x$ that has state set $\{m\}$ on all characters, and showing that all other species must have $x$ as a descendant, which forces any solution to this instance of the (P-)GCC-NB Problem to be a single-branch tree. We omit the details. 
As a corollary, we have that the $\{\{1\},\{2\},\{0,2\},\{0,1,2\}\}$-(P-)GCC-NB Problem is NP-complete. However, the complexity of the BKW case posed in [3] remains open. In the next subsections, we will show that in the BKW case the SB-GCC-NB Problem is polynomial-time solvable.

\section{The BKW Case of the SB-GCC-NB Problem Is Polynomial-Time Solvable}

First, we show that the $\{\{1\},\{0,2\}\}$-SB-GCC-NB and $\{\{1\},\{0,2\}\}$-P-GCC-NB Problems are polynomial-time solvable, by showing that they are equivalent to the Consecutive-Ones Property Problem 5, used in DNA physical mapping, interval graph recognition and data retrieval. The formal definition of this problem is as follows:

\section{Consecutive-Ones Property (C1P) Problem}

Input: An $n \times m$ 0-1-matrix $M$.

Question: Is there an order on the $m$ columns of $M$ in such a way that, for any row, the set of columns that have entry 1 in that row are consecutive in the order.

This property can be determined in linear time by building a $P Q$-tree $[5]$. We then build on the algorithm for the C1P Problem (namely, the algorithm for building a $P Q$-tree) to show that the $\{\{0\},\{1\},\{2\},\{0,2\}\}$-SB-GCC-NB Problem (the BKW Case of the SB-GCC-NB Problem) is also polynomial-time solvable.

Lemma 2. The $\{\{1\},\{0,2\}\}-S B-G C C-N B$ and $\{\{1\},\{0,2\}\}-P-G C C-N B$ Problems are polynomial-time solvable.

Proof. The solutions to the $\{\{1\},\{0,2\}\}$-SB-GCC-NB and $\{1\},\{0,2\}\}$-P-GCCNB Problems must fall on a single-branch tree and path, respectively. Because $T_{\alpha}$ is $0 \rightarrow 1 \rightarrow 2$ for any character $\hat{\alpha}$, all species where $\hat{\alpha}$ has state 1 must appear consecutively in this single-branch tree (path), otherwise there would be more than one transition from 0 to 1 in the phylogeny, for some character $\hat{\alpha}$. In this case of the SB-GCC-NB Problem, all other species can appear before (or after) this consecutive set of ones, because the "state-choosing" function $c$ can map these species to 0 (or 2). As such, this problem is exactly the problem of determining whether or not a 0 -1-matrix has the $\mathrm{C} 1 \mathrm{P}$, where each species is a column in this matrix. In this case of the P-GCC-NB Problem, if there does exist a solution $P$, then there is always a "state-choosing" function $c$ ' that reflects the fact that the corresponding matrix has the $\mathrm{C} 1 \mathrm{P}$. Therefore these cases are polynomial-time solvable.

We now consider the $\{\{0\},\{1\},\{2\},\{0,2\}\}$-SB-GCC-NB Problem, the BKW case of the SB-GCC-NB Problem. Here, for any character $\hat{\alpha}$, a species $s$ with $\alpha(s)=\{0,2\}$ can still appear before or after the consecutive set of ones 
(on this single-branch tree), however a species $s$ with $\alpha(s)=0$ has to appear before this set, while the species $s$ with $\alpha(s)=2$ has to appear after this set. So essentially, this is the problem of determining whether a 0-1-matrix has the $\mathrm{C} 1 \mathrm{P}$ again, however the matrix, in addition to containing zeros and ones, contains some special zeros, we call them $0^{-}\left(0^{+}\right)$, that must appear before (after) the set of consecutive ones of its row, in any consecutive-ones ordering. This case is thus equivalent to the following generalised version of the $\mathrm{C} 1 \mathrm{P}$ Problem:

\section{Extended Consecutive Ones (E-C1P) Property Problem}

Input: An $n \times m$ matrix $M$ with entries $0,1,0^{-}$or $0^{+}$.

Question: Is there an order on the $m$ columns of $M$ in such a way that, for any row, the set of columns that have entry 1 in that row are consecutive in the order, and any column that has entry $0^{-}\left(0^{+}\right)$in that row appears before (after) this consecutive set of ones.

Lemma 3. The E-C1P Problem is polynomial-time solvable.

Proof. We prove this by showing that a structure that encodes all solutions to the E-C1P problem can be built in polynomial-time. Given instance $M$ to the E-C1P Problem, we first construct $P Q$-tree $P Q_{M}$ for matrix $M$, where we have "forgotten" the labels of the special zeros (we treat $0^{-}$and $0^{+}$simply as 0 ). This can be done in $O(n+m)$-time [5]. It is clear that $P Q_{M}$ encodes a superset of the solutions to $M$. We then associate to each $P$ node, the empty partial order on its children, and to each $Q$ node, the set of directions \{left, right\}. Next, we obtain a list of order constraints imposed by the special zeros of $M$, by processing each pair of $0^{-}$and $1,0^{+}$and 1 , and $0^{-}$and $0^{+}$. For instance, if column $i$ has $0^{-}$and $j$ has 1 in some row $r$, then we add constraint $i<j$ to the list. We now update these sets that are associated with each $P$ and $Q$ node, one-by-one from the list, to incorporate these ordering constraints. The idea is that these sets will restrict the configurations each node in $P Q_{M}$ can have to the set of solutions of $M$.

When adding constraint $i<j$ from the list to $P Q_{M}$, we find the least common ancestor $a_{i, j}$ of $i$ and $j$ in $P Q_{M}$, which takes $O(m)$ steps:

- If $a_{i, j}$ is a $Q$ node, then we eliminate from its set, the direction that places $j$ before $i$. If the set of directions is now empty, then the algorithm halts, outputting that $M$ does not have a solution. This can be done in constant time.

- Otherwise $a_{i, j}$ is a $P$ node that stores some partial order on its children $\left\{v_{1}, \ldots, v_{k}\right\}=V$. First, we find the children of $a_{i, j}: v_{x}$ and $v_{y}$ such that the subtrees rooted at them contain $i$ and $j$, respectively. We add the constraint $v_{x}<v_{y}$ to the existing partial order at this $P$ node. If this constraint is not consistent with the existing partial order then the algorithm halts, outputting that $M$ does not have a solution. This partial order can be updated in time $O\left(k^{2}\right)$. Thus this step takes time $O\left(k^{2}\right) \subseteq O\left(m^{2}\right)$.

Since there are $O\left(\mathrm{~nm}^{2}\right)$ order constraints, and it takes time $O\left(\mathrm{~m}^{2}\right)$ to process each constraint, the algorithm takes time $O\left(\mathrm{~nm}^{4}\right)$.

Theorem 3. The BKW case of the SB-GCC-NB Problem is polynomial.

Proof. This follows from equivalence to the E-C1P Problem and Lemma 3 . 


\section{Conclusions and Open Problems}

We now conclude with a summary of results for the cases of the $\mathcal{Q}$-GCC-NB Problem, $\mathcal{Q} \subseteq\{\{0\},\{1\},\{2\},\{0,2\},\{0,1,2\}\}$, that are implied by our results, and what interesting cases are still open. This is conveyed in Fig. 1, where each row is a choice of $\mathcal{Q}$ for the $\mathcal{Q}$-GCC-NB Problem, and the columns specify whether the solution is restricted to (a) a single-branch tree, (b) a path and (c) a tree, respectively. In Sect. 3, Theorem 1 shows that the GCC-NB Problem remains hard even when the solution is restricted to be a single-branch tree (8a in Fig. 1). Note that this also gives entries 1a, 6a and 7a. Theorem 2 then gives entries $1 \mathrm{~b}, \mathrm{c}$ and $7 \mathrm{~b}, \mathrm{c}$. In Sect. 4, Lemma 2 gives entries $5 \mathrm{a}, \mathrm{b}$. Theorem 3 shows that the BKW Case of the SB-GCC-NB Problem is polynomial-time solvable, giving entries 2a, $3 \mathrm{a}$ and $4 \mathrm{a}$. Note, that the BKW Case of the GCC-NB Problem, posed in [3] still remains open (2c in Fig. 1). The cases marked with $(\dagger)$ follow from [32. In particular, notice that if $\mathcal{Q} \subseteq\{\{0\},\{1\},\{2\},\{0,1,2\}\}$, that is each element in $\mathcal{Q}$ is a directed path in $0 \rightarrow 1 \rightarrow 2$, then the $\mathcal{Q}$-GCC-NB Problem is polynomial-time solvable by [3]. Entries $13 \mathrm{a}, \mathrm{b}, \mathrm{c}$ involve only classical characters, and are thus polynomial-time solvable by [1]. The entries marked with $(\triangle)$ follow from our new preliminary results not included in this paper. In all remaining cases the problem becomes trivial as there always is a solution.

\begin{tabular}{|c|c|c|c|}
\hline $\mathcal{Q} \backslash$ soln & (a) branc & (b) path & (c) tree \\
\hline (1) $\{\{0\},\{1\},\{2\},\{0,2\},\{0,1,2\}\}$ & NP-c & NP-c & $\mathrm{NP}-\mathrm{c}^{\dagger}$ \\
\hline (2) $\{\{0\},\{1\},\{2\},\{0,2\}\}^{*}$ & $\mathrm{P}$ & NP-c $\triangle$ & $?$ \\
\hline (3) $\{\{0\},\{1\},\{0,2\}\}$ & $\overline{\mathrm{P}}$ & $\mathrm{NP}-\mathrm{c}^{\triangle}$ & $?$ \\
\hline (4) $\{\{1\},\{2\},\{0,2\}\}$ & $\mathrm{P}$ & $?$ & $?$ \\
\hline (5) $\{\{1\},\{0,2\}\}$ & $\bar{P}$ & $\overline{\mathrm{P}}$ & $?$ \\
\hline (6) $\{\{0\},\{1\},\{0,2\},\{0,1,2\}\}$ & NP-c & NP-c ${ }^{\square}$ & $\mathrm{NP}-\mathrm{c}^{\dagger}$ \\
\hline (7) $\{\{1\},\{2\},\{0,2\},\{0,1,2\}\}$ & NP-c & $\mathrm{NP}-\mathrm{c}$ & $\mathrm{NP}-\mathrm{c}^{1}$ \\
\hline (8) $\{\{1\},\{0,2\},\{0,1,2\}\}$ & $\mathrm{NP}-\mathrm{c}$ & $?$ & NP-c ${ }^{1}$ \\
\hline (9) $\{\{0\},\{2\},\{0,2\}\}(\cup\{\{0,1,2\}\}$ & $\mathrm{P}^{\triangle}$ & NP-c ${ }^{\triangle}$ & $\mathrm{P}^{\dagger}$ \\
\hline$(10)\{\{0\},\{1\},\{0,1,2\}\}(\cup\{\{2\}\})$ & $\mathrm{P} \triangle$ & NP-c $\triangle$ & $\mathrm{P}^{\dagger}$ \\
\hline (11) $\{\{0\},\{2\},\{0,1,2\}\}$ & $\mathrm{P}^{\triangle}$ & NP-c ${ }^{\triangle}$ & $\mathrm{P}^{\dagger}$ \\
\hline (12) $\{\{1\},\{2\},\{0,1,2\}\}$ & $\mathrm{P}^{\Delta}$ & NP-c $\triangle$ & $\mathrm{P}^{\dagger}$ \\
\hline$(13) \mathcal{Q} \subseteq\{\{0\},\{1\},\{2\}\}$ & $\mathrm{P}$ & $\mathrm{P}$ & $\mathrm{P}^{\dagger}$ \\
\hline
\end{tabular}

Fig. 1. Complexity of various cases of the GCC-NB Problem $(*)$ BKW Case $(\dagger)$ implied by [3]2] $(\triangle)$ new preliminary results

We now give comments on some of the cases that remain open. The $\{\{1\}$, $\{0,2\}\}$-SB-GCC-NB Problem (the solution must be a single-branch tree) corresponds naturally to the C1P Problem (the solution must be a total order). As such, the $\{\{1\},\{0,2\}\}$-GCC-NB Problem corresponds to a tree-C1P Problem which is defined as follows: Find a tree with vertex set containing the columns of a binary matrix $M$ and auxiliary columns such that for every row in $M^{\prime}$, a 
tree labelled by the row contracts to a tree $0-1-0$ after contracting any two vertices with the same label, where $M^{\prime}$ is a matrix containing $M$ and auxiliary columns. Similarly, one could define the tree-E-C1P Problem on matrices with $0^{-}, 0,0^{+}$and 1 . Now, determining the complexity of this tree-E-C1P Problem would provide an answer to the BKW Case of the GCC-NB Problem [3].

\section{References}

1. Agarwala, R., Fernandez-Baca, D.: A polynomial-time algorithm for the perfect phylogeny problem when the number of character states is fixed. SIAM J. on Computing 26(6), 1216-1224 (1994)

2. Benham, C.J., Kannan, S., Paterson, M., Warnow, T.: Hen's teeth and whale's feet: Generalized characters and their compatibility. Journal of Computational Biology 2(4), 515-525 (1995)

3. Benham, C.J., Kannan, S., Warnow, T.: Of chicken teeth and mouse eyes, or generalized character compatibility. In: Galil, Z., Ukkonen, E. (eds.) CPM 1995. LNCS, vol. 937, pp. 17-26. Springer, Heidelberg (1995)

4. Bodlaender, H., Fellows, M., Warnow, T.: Two strikes against perfect phylogeny. In: Kuich, W. (ed.) ICALP 1992. LNCS, vol. 623, pp. 273-283. Springer, Heidelberg (1992)

5. Booth, K.S., Lueker, G.S.: Testing for the consecutive ones property of, interval graphs, and graph planarity using PQ-tree algorithms. Journal of Computer and Systems Sciences 13(3), 335-379 (1976)

6. Felsenstein, J.: Numerical methods for inferring evolutionary trees. The Quarterly Review of Biology 57(4), 379-404 (1982)

7. Janis, C.: The sabertooth's repeat performances. Natural History 103, 78-82 (1994)

8. Kannan, S., Warnow, T.: Inferring evolutionary history from DNA sequences. SIAM J. on Computing 23(4), 713-737 (1994)

9. Kannan, S., Warnow, T.: A fast algorithm for the computation and enumeration of perfect phylogenies. In: SODA, pp. 595-603 (1995)

10. Kollar, E.J., Fisher, C.: Tooth induction in chick epithelium: Expression of quiescent genes for enamel synthesis. Science 207, 993-995 (1980)

11. McMorris, F.R., Warnow, T., Wimer, T.: Triangulating vertex colored graphs. SIAM J. on Discrete Mathematics 7(2), 296-306 (1994)

12. Opatrny, J.: Total ordering problem. SIAM J. on Computing 8(1), 111-114 (1979)

13. Steel, M.: The complexity of reconstructing trees from qualitative characters and subtrees. Journal of Classification 9, 91-116 (1992)

14. Telles, G.P., Meidanis, J.: Building PQR trees in almost-linear time. In: Proc. of GRACO, pp. 33-39 (2005) 